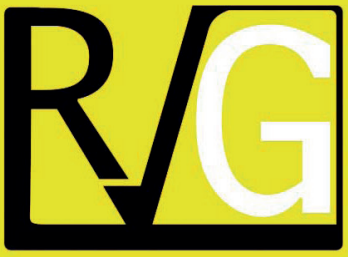

Julio - Septiembre, 2021

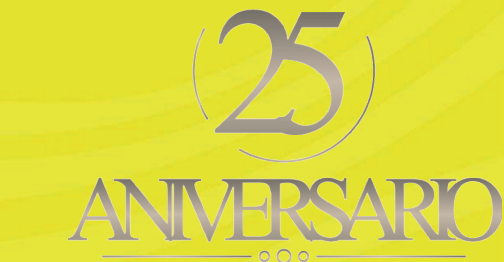

ANMERSARO
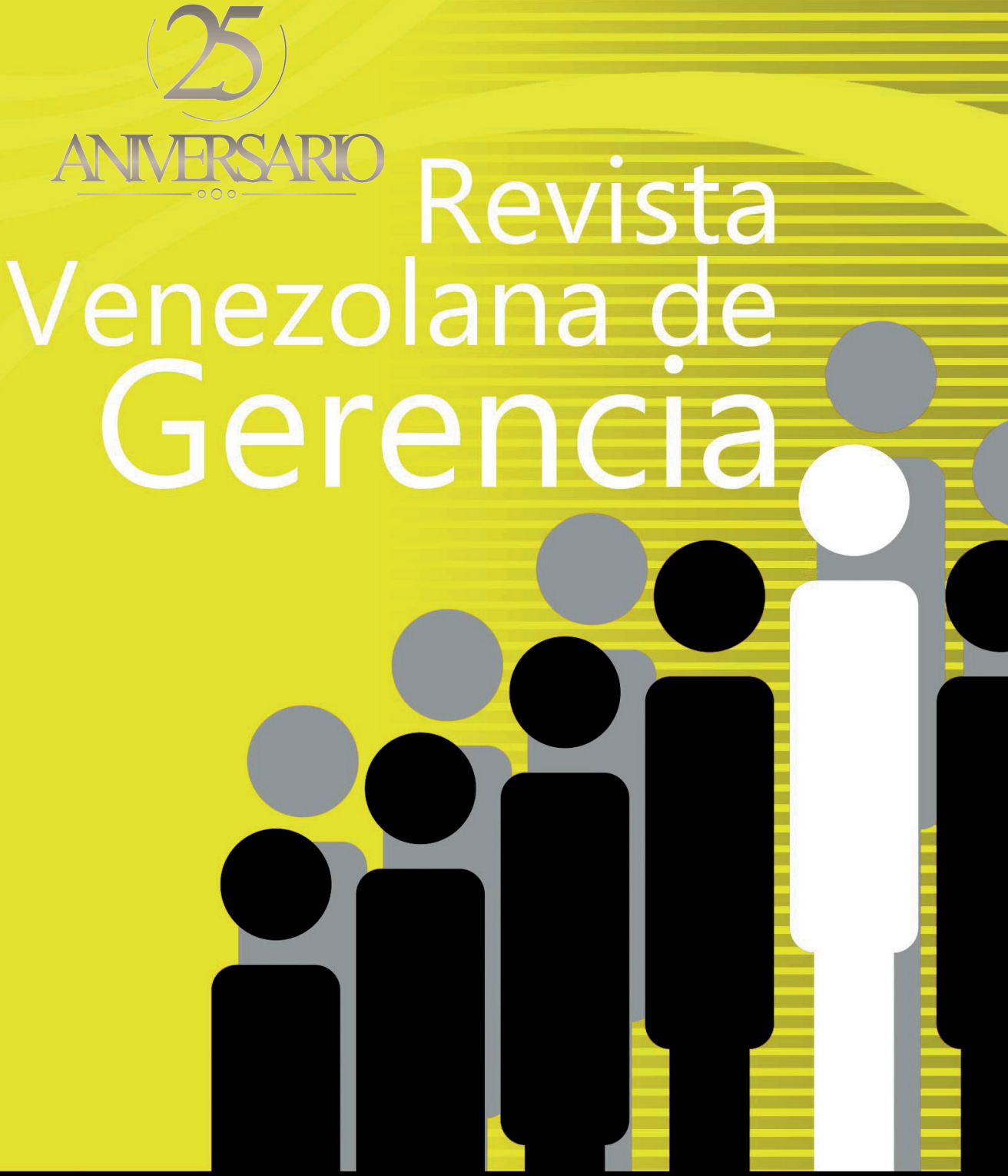

UNIVERSIDAD DEL ZULIA (LUZ)

Facultad de Ciencias Económicas y Sociales Centro de Estudios de la Empresa

ISSN 1315-99

Esta obra está bajo una licencia de Creative Comm Reconocimiento-NoComercial-Compartirlgual 3.0 Unpo http://creativecommons.org/licenses/by-nc-sa/3.0/deed.es 
COMO CITAR: Müller Pérez, J., Azuela Flores, J. I., y Jiménez Almaguer, K. P. (2021). Estrategias de descuento en el precio y de bonus pack en la intención de compra. Revista Venezolana de Gerencia (RVG), 26(95), 868-881. https://doi. org/10.52080/rvgluz.27.95.26
Universidad del Zulia (LUZ)

Revista Venezolana de Gerencia (RVG)

Año 26 No. 95 Julio-Septiembre 2021, 868-881

ISSN 1315-9984 / e-ISSN 2477-9423

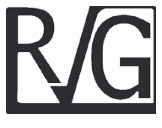

\title{
Estrategias de descuento en el precio y de bonus pack en la intención de compra
}

\author{
Müller Pérez Jessica* \\ Azuela Flores José Ignacio** \\ Jiménez Almaguer Karla Paola***
}

\section{Resumen}

Las promociones de marketing son prácticas ampliamente recurridas en el mercado. No obstante, existe una disyuntiva en los hallazgos sobre la efectividad de la estrategia de descuento y de la de bonus pack. Por lo anterior, el objetivo de esta investigación es analizar de manera comparativa el efecto de ambas estrategias en la intención de compra. Para ello se empleó un diseño experimental con 69 personas utilizando como producto una barra de chocolate, aplicando un análisis de la varianza (ANOVA) de un factor. Los resultados señalan que el descuento de precio tiene un efecto mayor en la intención de compra. Estos resultados contribuyen a comprender en mayor medida el comportamiento del consumidor. Asimismo, se destaca que es importante elegir la promoción adecuada para promover el consumo en el corto plazo de productos semejantes al empleado en este estudio.

Palabras clave: estrategias de precios; descuento en el precio; Bonus pack; intención de compra.

* Máster en administración de empresas por la Universidad Autónoma de Nuevo León. México. Doctorante del programa en Ciencias Administrativas de la Universidad Autónoma de Tamaulipas (UAT) y Profesora de horario libre de la Facultad de Comercio y Administración Victoria (UAT), México. Email: jsmuller@docentes. uat.edu.mx. ORCID: https://orcid.org/0000-0002-3212-9357

** Doctor por la Universidad de Oviedo (España), programa: Integración Económica, Competitividad y Entorno Institucional de la Empresa. Universidad Autónoma de Tamaulipas (UAT), México. Profesor Investigador en la Facultad de Comercio y Administración de Tampico de Empresas (UAT). iazuelaf@docentes.uat.edu.mx. ORCID: http://orcid.org/0000-0001-8084-9669

*** Doctora por la Universidad de Oviedo (España), programa: Integración Económica, Competitividad y Entorno Institucional de la Empresa. Universidad Autónoma de Tamaulipas (UAT). Profesora Investigadora en la Facultad de Comercio y Administración de Tampico de Empresas (UAT). kjimenez@docentes.uat.edu.mx; ¡imenez.karlap@gmail.com. ORCID: https://orcid.org/0000-0003-2464-3759 


\title{
Price discount strategies and bonus pack in purchase intention
}

\begin{abstract}
Marketing promotions are widely used practices. However, there is a dilemma on the effectiveness of the discount strategy and the bonus pack strategy. Therefore, the objective of this research is to compare the effect of both strategies on purchase intention. An experimental design was carried out with 69 people using a chocolate bar, applying a one-factor analysis of variance (ANOVA). The results indicate that the price discount has a greater effect on the purchase intention. These results contribute to a better understanding of consumer behavior. Likewise, it is highlighted that it is important to choose the appropriate promotion strategy to promote the consumption in the short term of products similar to the one used in this study.
\end{abstract}

Keywords: pricing strategies; price discount; Bonus pack; purchase intention.

\section{Introducción}

Los especialistas en marketing que desean realizar promociones tienen una variedad de opciones entre las que elegir. Los fabricantes de productos envasados gastan una proporción significativa de sus presupuestos de marketing en promociones de ventas (McColl et al. 2020). A pesar de los amplios recursos asignados a las promociones de ventas, los gerentes a menudo no están seguros de qué combinaciones de atributos de promoción y en qué circunstancias, serán más efectivas para lograr sus objetivos de marketing (Peng et al. 2020).

Las estrategias de precio, ya sea mediante cambios al precio o a la cantidad de producto ofrecido, son una práctica muy extendida en el mercado. Los descuentos al precio han sido la estrategia dominante para estimular la compra (Hardesty \& Bearden, 2003: 17); no obstante, la estrategia Bonus pack, consiste en recibir más o un excedente de un producto por el mismo precio (Mishra \& Mishra, 2011: 197), estrategia que ha ido incrementando su popularidad en diversas partes del mundo. De hecho, la evidencia empírica y la información de encuestas aplicadas en los Estados Unidos revela el creciente uso de Bonus pack como estrategia de precios para una amplia variedad de categorías de productos incluidos ropa (compra uno y lleva el segundo gratis), alimentos y artículos para el hogar (por ejemplo: pastas dentales, productos para el cuidado del cabello, baterías, entre otros) (Chen et al. 2012).

La investigación previa sobre estas estrategias de precio revela que los consumidores prefieren Bonus pack porque éste es asociado con una ganancia (más cantidad gratis); mientras que la reducción en el precio es percibida como una reducción dentro de una perdida (Chen et al. 2012:10; Diamond \& Sanyal, 1990). Esta preferencia por las estrategias Bonus pack prevalece incluso cuando los consumidores debieran mostrarse 
indiferentes; es decir, cuando se trata del mismo descuento, pero expresado de distinta forma. De tal modo que, diferentes representaciones del mismo descuento en precio no conducen a la misma respuesta de los consumidores (Heath et al. 1995: 93).

Hallazgos más recientes han demostrado que la percepción que tiene el consumidor hacia las ofertas dependerá del grado en que los consumidores realicen cálculos con precisión sobre los descuentos y los precios finales de compra (Estelami, 2003:10; Kim \& Kramer, 2006:319). En general, los Precios de Descuento dificultan a los consumidores calcular los descuentos y, consecuentemente, estimar correctamente el precio final (Estelami, 2003: 10; Morwitz et al. 1998:459).

Como se señala previamente, existe una disyuntiva en los hallazgos sobre la efectividad de la estrategia de descuento y de la de bonus pack. De ahí la importancia de estudiar la forma en que los consumidores deducen un descuento en el precio y si ésta varía en función del formato presentado (Precio de Descuento o Bonus pack). El objetivo de este trabajo es analizar de manera comparativa el efecto de ambas estrategias en la intención de compra. Para lograr el objetivo se empleó un diseño experimental que incluyó tres escenarios: 1) Precio de Descuento, 2) descuento Bonus pack y 3) Precio de Referencia que fungió como grupo de control. Para analizar los efectos de las anteriores estrategias de precio sobre la intención de compra, en congruencia con la investigación previa (Mishra \& Mishra, 2011:198; Xu \& Huang, 2014:1298), se utilizó el chocolate. Se aplicó un análisis de la varianza (ANOVA) de un factor.

\section{Bonus pack versus Precios de Descuento}

Los minoristas utilizan estrategias promocionales, como los productos destacados, los productos en oferta y los obsequios, para aumentar las ventas y las ganancias en mercados diversos (Andrade et al. 2010; ChinchayVillarreyes et al. 2020; Choi \& Chen, 2019; Guerreiro et al. 2009; McCabe \& Branco, 2019; Valencia et al. 2019). Sin embargo, los descuentos en los precios y los paquetes de bonificación (bonus pack) son las estrategias de promoción de ventas más empleadas (Chen et al. 2012).

La premisa que subyace en la literatura previa sugiere que los consumidores, frente a los Precios de Descuento, se inclinan más hacia las estrategias de precio Bonus pack (Chandran \& Morwitz, 2006: 390; Chen et al. 2012:10; Diamond \& Sanyal, 1990). De acuerdo con estos autores, este resultado obedece a una idea muy sencilla: ganar o perder. Diamond y Sanyal (1990) señalaron que las estrategias de bonificación en producto (Bonus pack), son percibidas por los consumidores como "ganancias" mientras que los precios de descuento son vistos como "reducciones de las pérdidas". Consecuentemente, las estrategias Bonus pack se prefieren por encima de los precio de descuento, puesto que una "ganancia" en cantidad es preferida antes que una reducción en las "perdidas" monetarias (Chen et al. 2012: 2).

La ambigüedad del valor monetario asociado a las bonificaciones destaca como otra explicación de la preferencia hacia Bonus pack. Los consumidores evalúan las bonificaciones como una parte independiente del precio (es decir, 
como algo gratis, algo adicional), lo que los conduce a valorar más las estrategias de bonificación que las de Precio de Descuento (Chandran \& Morwitz, 2006: 390-391).

Finalmente, la dificultad para interpretar cambios expresados en porcentajes es otra explicación de la preferencia de Bonus pack por encima de los Precios de Descuento. Los consumidores procesan erróneamente la información cuando está expresada en porcentajes; lo cual los conduce a sesgos (Chen et al. 2012: 3). Este sesgo se magnifica cuando se trata de estrategias mixtas en las que se combinan bonificación en producto (Bonus pack) y deducciones en el precio. Por ejemplo, Chen y Rao (2007: 335) encontraron que los consumidores muestran mayor preferencia por deducciones dobles/mixtas: $25 \%$ más (en bonificación de producto) más un adicional del $20 \%$ de descuento; que por su equivalente descuento en precio de $40 \%{ }^{1}$. Los consumidores tienden a sumar las expresiones porcentuales incluso cuando estas no dependen del mismo valor base (es decir, bases expresadas en distintas unidades de medida: producto y precio). Después de todo $y$, en percepción del consumidor, una promoción del $45 \%$ es mayor que una de $40 \%$.

Sin embargo, no toda la evidencia empírica ha registrado un predominio de Bonus pack (Vasić Nikcević et al. 2020). Por ejemplo, Smith y Sinha (2000: 88) encontraron resultados mixtos dependiendo de si el producto era caro o barato. Así pues, identificaron mayor preferencia por los Precios de Descuento para los productos caros pero mayor preferencia por Bonus pack cuando los productos son baratos.

Asimismo, se ha identificado que el impacto de Bonus pack y Precio de Descuento varía en función de la magnitud de la promoción/descuento. Es decir, dependiendo de si la promoción/ descuento es baja (10\%), moderada $(25 \%)$ o alta (50\%). Hardesty y Bearden (2003: 19) identificaron que en promociones/descuentos tanto bajos como moderados, los consumidores se mostraron indiferentes tanto a los Precios de Descuento como a Bonus pack. Sin embargo, en promociones/descuentos de alta magnitud (descuentos del 50\%), los consumidores prefirieron Precios de Descuento.

El impacto de estas estrategias de precio también varía en función de la categoría de producto; por ejemplo, si se trata de productos sanos o nocivos a la salud; o si se trata de productos perecederos o no perecederos (Petit et al. 2020). Mishra y Mishra (2011: 198) analizaron la influencia de las estrategias basadas en precio (Precios de Descuento) y cantidad (Bonus pack) sobre el consumo de alimentos saludables (virtue) y no saludables (vice). Los autores encontraron que los consumidores prefieren estrategias de precio Bonus pack por encima de Precios de Descuento cuando se trata de alimentos saludables. Sin embargo, las preferencias por las estrategias de precio cambian cuando se trata de productos no saludables. En estos casos, los Precios de Descuento destacan por

$25 \%$ de bonificación en producto es equivalente a $20 \%$ de descuento en precio. 
encima de Bonus pack.

De acuerdo con los autores, la sensación de culpa juega un papel relevante impidiendo a los consumidores generar una buena justificación para comprar este tipo de productos en Bonus pack considerando que hacerlo supondría consumir más cantidad de un producto que no favorece a la salud (Huyghe y Van Kerckhove, 2013). Por su parte, Smith y Sinha (2000: 88) evaluaron el impacto de estas estrategias identificando que, los Precios de Descuento logran mayor preferencia cuando se trata de productos perecederos.

Finalmente, el efecto de las estrategias en la intención de compra también se modifica en función de qué tan familiarizado esté el consumidor con el producto. De tal modo que, los consumidores prefieren precios de descuento cuando se trata de productos con los que no están familiarizados, por ejemplo, cuando se trata de productos de marcas que recientemente han entrado al mercado (marcas nuevas), la evidencia previa revela que los consumidores muestran mayor preferencia hacia los precios de descuento. Pero se muestran indiferentes al tipo de estrategia de precio (Precio de Descuento o Bonus pack) cuando se trata de productos con los que están familiarizados (Chen et al. 2012: 10).

$$
\text { Una explicación alternativa }
$$
sobre la subvaloración de los precios de descuento está relacionada con los cálculos que involucra estimar el descuento. Estelami, (2003: 10) y Morwitz et al (1998: 459) identificaron que, en general, los precios de descuento dificultan a los consumidores calcular los descuentos $\mathrm{y}$, consecuentemente, estimar correctamente el precio final. Frente a una estrategia de Precios de Descuento los consumidores tienen que realizar operaciones para poder evaluar el precio final (por ejemplo: $\$ 99$ - $\$ 99$ x $20 \%$ de descuento) lo que supone hacer dos operaciones que, en muchos casos, los consumidores no están dispuestos a realizar conduciendo a un mal procesamiento de la información en los precios de descuento y, finalmente, a una subvaloración de esta estrategia frente a otras estrategias de precio (Morwitz et al. 1998: 459).

Por su parte, Kim y Kramer (2006: 316) identificaron que la valoración de los precios de descuento aumenta cuando se emplean formas novedosas de descuento. Concretamente, los autores identificaron que Precios de Descuento Novedosos como: "paga el $60 \%$ del precio regular", frente a las presentaciones tradicionales de Precio de Descuento ("obtén un 40\% de descuento sobre el precio regular") incrementan la intención de compra de los consumidores ${ }^{2}$.

Como puede percibirse a partir de la literatura antes mencionada, son diversos los aspectos que tienen un rol importante en las distintas estrategias de precios: ambigüedad en el valor monetario, precio alto o bajo del producto, nivel del porcentaje o valor, si

2 Note que en la estrategia de descuento novedosa los consumidores sólo tienen que hacer una operación aritmética (Precio Regular x 60\%= Precio Final) mientras que en las estratégicas de precio de descuento tradicionales los consumidores tienen que hacer dos operaciones (Precio Regular - Precio Regular x 40\%= Precio Final). 
es un bien perecedero o no, si se está familiarizado con el producto, la dificultad del cálculo y lo novedoso del beneficio. Asimismo, la revisión previa de literatura indica que son más los estudios que muestran la preferencia por la estrategia de descuento que la de bonus pack.

En resumen, las estrategias de precios de descuento incrementan la intención de compra cuando estas se utilizan en productos en los que un aumento en el consumo no es bueno para la salud (como el caso de productos hedónicos como el chocolate; o productos nocivos para la salud como los cigarros y el alcohol) y en productos caros. De hecho, un plan de promoción de ventas puede ser más efectivo cuando está orientado al beneficio hedónico en el caso de productos alimenticios (Sinha \& Verma, 2020)

Asimismo, estas estrategias muestran mejor desempeño cuando se trata de productos perecederos, con los que los consumidores están familiarizados $\mathrm{y}$, finalmente, cuando se usan estrategias de descuento novedosas. Del mismo modo, se prefiere por los consumidores la estrategia de descuento cuando se trata de descuentos altos (50\% o más). Por ello, aunque existe divergencia en la literatura empírica analizada aquí entre la preferencia de una estrategia u otra, resulta evidente que se preferirá la estrategia de precios con descuento frente a la de bonus pack.

Con base en lo anterior se propone la siguiente hipótesis:

H1: Las estrategias de Precio de Descuento Novedoso (vs. Bonus pack) incrementan la intención de compra en productos hedónicos.

\section{Consideraciones metodológicas del estudio}

En este apartado se aborda la metodología, los participantes, el procedimiento y las medidas empleadas en contraste de la hipótesis previamente desarrollada. Para la recopilación de la información se llevó a cabo un experimento de laboratorio que incluyó tres escenarios: 1) descuento porcentual, 2) descuento Bonus pack y 3) grupo de control; que representan dos distintas estrategias de precios y un grupo de control. Para analizar los efectos de las anteriores estrategias de precio sobre la intención de compra, en congruencia con investigación previa (Mishra \& Mishra, 2011:198; Xu \& Huang, 2014:1298), se utilizó el chocolate. Concretamente, y nuevamente en congruencia con la literatura, se eligió una barra de chocolate con leche de la marca Hershey's ${ }^{3}$ de 40 grs., una marca con la que están familiarizados los participantes (Chen et al. 2012: 10), de un precio promedio en el mercado de $\$ 15.50$ pesos mexicanos. Se optó por un solo producto para que los participantes se centrarán en la estrategia de precios más que en diversos productos. Asimismo, se optó por un producto hedónico con el propósito de contribuir a la literatura que ha mostrado evidencia que favorece la estrategia de la que se parte la comparativa aquí planteada $(\mathrm{H} 1)$ (descuento de precios).

Con el propósito de evitar sesgos en la asignación, los participantes fueron asignados aleatoriamente a uno de los

3 Con un $62 \%$ de participación en el mercado nacional, Hershey's destaca como la marca de chocolate más consumida entre los mexicanos (EI Financiero, 2017), y la versión que más se consume es el chocolate con leche (FEEBBO, 2018). 
tres escenarios posibles.

Este estudio contribuye a la literatura previa al utilizar para la comparativa un producto que posee varias características mencionadas en estudios previos: precio bajo, nivel alto de descuento o producto adicional, perecedero, no saludable y familiar.

Los participantes fueron estudiantes, todos ellos cursando activamente licenciaturas de negocios (administración, economía, tecnologías de la información y contabilidad). El criterio de selección empleado fue el muestreo no probabilístico. Se recurrió a estudiantes universitarios de licenciatura y posgrado de una dependencia de una universidad pública del Estado de Tamaulipas (México). La muestra final constó de 69 personas, de ellas $48 \%$ son mujeres y $52 \%$ son hombres de edades entre 18 y 29 años. El uso de estudiantes como sujetos de análisis ha sido cuestionado y considerado como una fuente potencial de sesgo. No obstante, Exadaktylos et al. (2013:4) han demostrado que estos no se comportan diferente de una población compuesta por participantes distintos a estudiantes (no-estudiantes). De hecho, Höst, Regnell y Wohlin (2000) identificaron que el uso de muestras compuestas por estudiantes universitarios conduce a resultados precisos dentro de los estudios de análisis causal.

Por otra parte, el uso de muestras conformadas por estudiantes tiene amplias ventajas; una de ellas es la relacionada con la homogeneidad. Se ha identificado que los estudiantes constituyen un grupo homogéneo que conduce a predicciones teóricas de mayor precisión que si se utilizaran muestras heterogéneas (Calder et al. 1981:200); otra ventaja es el nivel de educación en el cual desarrollan la habilidad numérica para interpretar correctamente los porcentajes presentados en las diferentes estrategias de precios (Purnhagen \& van Herpen, 2017: 229).

Finalmente, la conveniencia de esta muestra también se ve reflejada cuando se compara con el segmento con mayor consumo de chocolate en México. De acuerdo con los datos de Kantar World Panel (2017) las personas (principalmente las mujeres) con edades comprendidas entre los 18 y 25 años son quienes compran chocolate con mayor frecuencia.

Para evaluar los efectos de las estrategias de precios se diseñaron tres condiciones:

i. Escenario Descuento Novedoso: éste representó un descuento directo sobre el precio de mercado, expresado en términos porcentuales (Precios de Descuento). A diferencia de los mecanismos de descuento tradicionales en los que el descuento se presenta como una deducción porcentual sobre el precio de mercado $(20 \%$ de descuento); en esta investigación se emplea una alternativa novedosa en la que el descuento se presenta como una proporción de lo que se debe pagar sobre el precio regular (paga solo el $83.33 \%$ de su precio original)

ii. Escenario Bonus pack: expresó el descuento en forma de Bonus pack, bajo esta condición el descuento en el precio se ve reflejado en una mayor cantidad de producto: 20\% más; ofreciendo $48 \mathrm{~g}$. de chocolate sobre una base de 40 gramos por el mismo precio (precio regular de \$15.50).

iii. Grupo de Control: Finalmente: en este escenario se presentó el estímulo sin descuentos en el precio. Es decir, se presentó la barra de chocolate Hershey's de 40 grs a un precio de 
15.50. Los miembros de este grupo serán tratados como grupo de control.

En las dos condiciones experimentales (Descuento Novedoso y Bonus pack) se empleó un descuento equivalente al $20 \%$ del precio de mercado. La selección de este descuento fue con base en Gupta y Cooper (1992: 409) quienes señalan que, para atraer la atención de los consumidores, un descuento debe ser superior al $15 \%$. En lo relativo al precio de referencia empleado: \$15.50, este se ajustó a los precios de mercado.

Los participantes fueron asignados aleatoriamente y en la misma proporción (23) a cada uno de los escenarios antes descritos. A los participantes se les mencionó que formaban parte de un estudio cuyo objetivo era el de analizar sus gustos por el chocolate, con el propósito de no influir en sus respuestas. En todos los escenarios, los participantes fueron situados frente a una computadora a través de la que se llevó a cabo el experimento. Luego de ubicar a los participantes y proporcionarles las instrucciones generales, el protocolo continuó a través de una página electrónica. Cada participante recibió instrucciones a través de la página web. Posteriormente se les presentó un folleto promocional de una tienda de autoservicio anunciando una barra de chocolate con el precio regular (precio de referencia) al que se ofrece en el mercado. Para los miembros del escenario Descuento Novedoso, el protocolo continuó mostrándoles una segunda imagen en la que se incluía la misma barra de chocolate con la aplicación del descuento novedoso "paga el 83.33\% del precio regular"

Por su parte, para los integrantes del escenario Bonus pack, luego del precio de referencia, el protocolo continuó con una segunda imagen en la que expuso un paquete de bonificación (Bonus pack) ofreciendo $48 \mathrm{~g}$. de chocolate a precio regular de $\$ 15.50$ (anteriormente 40g. por \$15.50) (Imagen $2)$. Mientras que a los integrantes del grupo de control sólo se les mostró la imagen con el precio de referencia. Luego de presentar los estímulos, el protocolo finalizó solicitando a los participantes que registran su intención de compra a través de una escala de intención.

Para medir la intención de compra se adecuó la escala propuesta por Kim y Kramer (2006: 315). La escala se constituyó de un solo ítem que mide la probabilidad de adquirir el chocolate: "¿qué tan probable es que compres el chocolate del anuncio?". Para registrar la intención se utilizó una escala Likert de 7 puntos en donde: $1=$ muy improbable y $7=$ muy probable. Si bien, la medición de una variable latente (como lo es la intención de compra) a través de un único ítem puede ser cuestionable, la literatura previa ha demostrado que, en el caso concreto de la intención de compra, está puede ser medida a través de un solo indicador observable (Hayduk \& Glaser, 2000: 12), manteniendo la misma validez predictiva que en el uso de múltiples mediciones (Bergkvist, 2015: 252) y favoreciendo en la reducción de costos (Ang \& Eisend, 2018: 223).

\section{Estrategias de precio sobre la intención de compra: Resultados}

Para analizar los efectos de las estrategias de precio (Descuento Novedoso y Bunus Pack) sobre la intención de compra, se llevó a cabo un análisis de la varianza (ANOVA) de un 
factor.

Los resultados muestran que hay un efecto significativo de las estrategias de precios sobre la intención de compra $(F=5.93 ; p=.004)$. Como se muestra en la Tabla 1, en promedio la intención de compra es mayor cuando se emplean estrategias de descuento novedosas (media $=5.43)$ y, como cabría esperar, menor en el caso de los precios de referencia [grupo de control; media= 3.78].

\begin{tabular}{|c|c|c|}
\hline \multicolumn{3}{|c|}{ Intención de Compra } \\
\hline Estrategias de Precio & Media & $\begin{array}{l}\text { Desviación } \\
\text { Estándar }\end{array}$ \\
\hline $\begin{array}{l}\text { Precio de referencia (grupo } \\
\text { de control) }\end{array}$ & 3.78 & 2.06 \\
\hline Bonus pack & 4.43 & 1.59 \\
\hline $\begin{array}{l}\text { Precio de descuento } \\
\text { (descuento novedoso) }\end{array}$ & 5.43 & 1.12 \\
\hline & \multicolumn{2}{|c|}{$\begin{array}{l}F=5.93 \\
p=.004 \\
F d e \text { Welch }=6.85 \\
p=.003\end{array}$} \\
\hline
\end{tabular}

Fuente: elaboración propia

Los contrastes planeados revelan que, tal y como se esperaba, las estrategias de precio ya sea Bonus pack o Descuento Novel incrementan significativamente la intención de compra respecto al grupo de control en donde no se ejerce ninguna promoción, $t(31.965)=2.420, p=.021$. Asimismo, y en congruencia con la hipótesis propuesta en esta investigación, los resultados de los contrates muestran que las estrategias de precio Descuento Novel incrementan significativamente la intención de compra, t $(39.534)=2.465$, $p=.018$ (Tabla 2).

Tabla 2

Pruebas de contrastes

\begin{tabular}{llll}
\hline Contrastes & $t$ & $g l$ & $p$ \\
\hline Precio de referencia/g. control VS. Bonus pack; Precio de Descuento & 2.42 & 31.96 & .021 \\
\hline Bonus pack VS. Precio de Descuento & 2.46 & 39.53 & .018 \\
\hline
\end{tabular}

Fuente: elaboración propia

En resumen, en este caso, no se rechaza la hipótesis planteada en donde se propone que la presentación de un precio de descuento novedoso aumenta la intención de compra del consumidor en productos hedónicos (chocolate). Además, cabe añadir que la prevalencia de las promociones de precios es muy 
específica del contexto (Hawaldar et al. 2019; Kaur et al. 2020). Cabe recordar que los resultados de todo experimento requieren replicarse para poder generalizar los mismos. Estos resultados son congruentes con los alcanzados en Kim y Kramer (2006: 315), Mishra y Mishra (2011: 200), Smith y Sinha (2000: 88) y Hardesty y Bearden (2003); el impacto de los Precios de Descuento es superior a Bonus pack cuando se trata de productos que no favorecen a la salud como es el caso del chocolate. En estos casos la sensación de culpa o arrepentimiento puede jugar un papel relevante impidiendo a los consumidores generar una buena justificación para comprar este tipo de productos en Bonus pack considerando que hacerlo supondría consumir más cantidad de un producto que no favorece a la salud (Mishra y Mishra, 2011: 200).

Asimismo, los resultados de este trabajo concuerdan con los obtenidos por Chen et al. (2012:10) quienes señalan que las estrategias de Precio de Descuento tienen mayor impacto cuando se trata de productos/marcas con las que los consumidores están familiarizados. En congruencia con lo anterior, los resultados de este trabajo muestran mayor impacto de los Precios de Descuento sobre la intención de compra de chocolates de una reconocida marca multinacional que ostenta la mayor cuota en los mercados de EE.UU ${ }^{4}$ y México ${ }^{5}$. Finalmente, estos resultados también son congruentes con los alcanzados por Kim y Kramer (2006: 315) quienes, como en esta investigación, mostraron que estrategias de Precio de Descuento presentadas de una forma novedosa como: paga el $80 \%$ de precio regular, frente a las estrategias Bonus pack, tiene mayor impacto motivando la intención de compra.

Los resultados de esta investigación muestran que, tal y como se propuso, la presentación de precios de descuento aumenta la probabilidad de adquirir un producto hedónico y económico (Park, 2016; Xu \& Huang, 2014) frente a la de Bonus pack. Estos hallazgos concuerdan con literatura 4

De acuerdo con STATISTA (2015) Hershey's fue la empresa de chocolates con mayor cuota de mercado en los EE UU con un $44 \%$. previa.

Los resultados de este trabajo conllevan algunas implicaciones teóricas y prácticas. En primer lugar, lo hallado en esta investigación concuerda con una corriente de la literatura. Asimismo, al analizar un alimento hedónico se contribuye a la comprensión del comportamiento del consumidor en este tipo de productos y se encuentra evidencia que da indicios que guían la elección de estrategias de promoción en este tipo de productos y marca en particular. En tercer lugar, dentro del marketing mix, la promoción de ventas es una de las de mayor impacto en el consumo en el corto plazo, especialmente en compras por impulso. De ahí que sea necesario analizar con detenimiento las

4 De acuerdo con STATISTA (2015) Hershey's fue la empresa de chocolates con mayor cuota de mercado en los EE UU con un $44 \%$.

5 De acuerdo con el periódico El Universal (2017) México es la operación más rentable para la estadounidense The Hershey Company. Del $90 \%$ de la producción en México es para consumo local y el $10 \%$ se envía al mercado estadounidense y menos del $1 \%$ a América Latina. 
estrategias posibles para promover de manera eficiente las ventas de productos como el aquí estudiado.

\section{Conclusiones}

Las promociones de marketing en forma de cambios en precios y cantidad son muy comunes en el mercado. El conocer cómo los clientes recopilan y evalúan los cambios en la información sobre porcentajes y los emplean para tomar decisiones es de gran importancia para los investigadores y los profesionales del marketing. Con el propósito de analizar de manera comparativa el efecto de la estrategia de descuento y de la estrategia bonus pack en la intención de compra se realizó un experimento para conocer si los grupos creados diferían entre sí.

En este sentido, se pudo constatar que el precio de descuento es la estrategia más efectiva en comparación con el bonus pack, ya que el precio de descuento hace percibir al consumidor que está ahorrando dinero $\mathrm{y}$, al mismo tiempo, no está consumiendo un producto que puede llegar a hacerle sentir culpa si lo consume en exceso. Además, la presentación de un Descuento Novedoso "paga el $\mathrm{X} \%$ del precio regular" aumenta la percepción de profundidad de descuento $y$, por lo tanto, aumenta las intenciones de compra.

Cabe mencionar que estos resultados presentan algunas limitantes. Por un lado, se analiza un solo tipo de producto (alimento, hedónico y económico). Igualmente, solo se abordó a estudiantes universitarios, por lo que los hallazgos no se pueden generalizar hasta repetir este estudio, lo cual es una característica de los experimentos. Del mismo modo, únicamente se manipuló una cantidad de descuento y una de bonus pack, por lo que los resultados deben interpretarse con precaución. Futuras investigaciones pueden hacer comparaciones distintas considerando diferentes tipos de productos, alimentos, porcentajes, cantidades de Bonus pack y contrastar las características de los participantes ampliando el tipo de participantes con base en el producto bajo estudio.

\section{Referencias bibliográficos}

Andrade, C., Fucci, M., \& Morales, M. (2010). Estrategias de marketing promocional en unidades de servicio de información de la Universidad del Zulia. Revista de Ciencias Sociales, 16(1), 68-79. https://doi. org/10.31876/rcs.v16i1.25485

Ang, L., \& Eisend, M. (2018). Single versus multiple measurement of attitudes: A meta-analysis of advertising studies validates the single-item measure approach. Journal of Advertising Research. https://doi.org/10.2501/JAR-2017001

Bergkvist, L. (2015). Appropriate use of single-item measures is here to stay. Marketing Letters, 26(3), 245-255. https://doi.org/10.1007/s11002-0149325-y

Calder, B., Phillips, L., y Tybout, A. (1981). Designing Research for Application. Journal of Consumer Research, 8(2), 197-207. https://econpapers.repec. org/article/oupjconrs/v 3a8 3ay 3a 1981 3ai 3a2 3ap 3a197-207.htm

Carlson, J. P. (2018). Consumer evaluations of bonus packs offered with price discounts. Journal of Consumer Marketing, 35(1), 22-31. https://doi.org/10.1108/JCM-092015-1555

Chandran, S., \& Morwitz, V. G. (2006). 
The Price of "Free"-dom: Consumer Sensitivity to Promotions with Negative Contextual Influences. Journal of Consumer Research, 33(3), 384-392. https://doi. org/10.1086/508439

Chen, H. A., Marmorstein, H., Tsiros, M., \& Rao, A. R. (2012). When more is less: The impact of base value neglect on consumer preferences for bonus packs over price discounts. Journal of Marketing, 76(4), 64-77. https://doi.org/10.1509/jm.10.0443

Chen, H., \& Rao, A. R. (2007). When two plus two is not equal to four: Errors in processing multiple percentage changes. Journal of Consumer Research, 34(3), 327-340. https:// doi.org/10.1086/518531

Chinchay-Villarreyes, S. S., Cango Córdova, J. I., Aldana Tume, A. A., \& Seminario Sanz, R. S. (2020). Estrategias de promoción para el fomento del turismo religioso en Perú/ Promotion strategies for the promotion of religious tourism in Peru. Revista de Ciencias Sociales, XXVI. https://doi.org/10.31876/rcs. v26i3.33247

Choi, H. S., \& Chen, C. (2019). Archived version from NCDOCKS Institutional Repository The Effects Of Discount Pricing And Bundling On The Sales Of Game As A Service: An Empirical Investigation. The effects of discount pricing and bundling on the sales of game as a service: an empirical investigation. 21-Journal of Electronic Commerce Research, 20(1). https:// bit.ly/3jirk9Q0

Diamond, W. D., \& Sanyal, \&gt;Abhijit. (1990). The Effect of Framing on the Choice of Supermarket Coupons. ACR North American Advances, NA-17. https://www.acrwebsite.org/ volumes/7055/volumes/v17/NA-17/ full
El Financiero (2017). ¿Cuál es el chocolate 'favorito' de México? https://www.elfinanciero.com.mx/ empresas/cual-es-el-chocolatefavorito-de-mexico

Estelami, H. (2003). The Effect of Price Presentation Tactics on Consumer Evaluation Effort of Multi-Dimensional Prices. Journal of Marketing Theory and Practice. https://doi.org/10.1080 /10696679.2003.11501934

Exadaktylos, F., Espín, A. M., \& BrañasGarza, P. (2013). Experimental subjects are not different. Scientific Reports, 3(1), 1-6. https://doi. org/10.1038/srep01213

Farrag, D. A. (2017). Impact of Shari'ah on Consumers' Behavior Toward Sales Promotion Tools: Focus on Egyptian Convenience Products. Journal of Food Products Marketing, 23(5), 533-552. https://doi.org/10.10 $\underline{80 / 10454446.2015 .1048020}$

FEEBBO. (2018). Estudio de mercado sobre el consumo del chocolate I Feebbo Mexico. http://blog. feebbomexico.com/estudio-demercado-sobre-el-consumo-delchocolate/

Guerreiro, R., Gouvêa, M. A., \& Dos Santos, A. (2009). Strategic and economic issues regarding to bonus quantity policy. RAE Revista de Administracao de Empresas, 49(1), 49-61. https://doi.org/10.1590/ $\underline{\mathrm{S} 0034-75902009000100007}$

Gupta, S., \& Cooper, L. G. (1992). The Discounting of Discounts and Promotion Thresholds. Journal of Consumer Research. https://doi. org/10.1086/209310

Hardesty, D. M., \& Bearden, W. O. (2003). Consumer evaluations of different promotion types and price presentations: The moderating role of promotional benefit level. Journal 
Müller Pérez Jessica, Azuela Flores José Ignacio, Jiménez Almaguer Karla Paola Estrategias de descuento en el precio y de bonus pack en la intención de

compra

of Retailing, 79(1), 17-25. https://doi. org/10.1016/S0022-4359(03)00004$\underline{6}$

Hawaldar, I. T., Ullal, M. S., Birau, F. R., \& Spulbar, C. M. (2019). Trapping fake discounts as drivers of real revenues and their impact on consumer's behavior in India: A case study. Sustainability (Switzerland), 11(17). https://doi.org/10.3390/su11174637

Hayduk, L. A., \& Glaser, D. N. (2000). Jiving the Four-Step, Waltzing Around Factor Analysis, and Other Serious Fun. Structural Equation Modeling, 7(1), 1-35. https://doi.org/10.1207/ S15328007SEM0701 01

Heath, T. B., Chatterjee, S., \& France, K. R. (1995). Mental Accounting and Changes in Price: The Frame Dependence of Reference Dependence. Journal of Consumer Research, 22(1), 90. https://doi. org/10.1086/209437

Höst, M., Regnell, B., \& Wohlin, C. (2000). Using students as subjects - a comparative study of students and professionals in lead-time impact assessment. Empirical Software Engineering. https://doi. org/10.1023/A:1026586415054

Huyghe, E., \& Van Kerckhove, A. (2013). Can fat taxes and package size restrictions stimulate healthy food choices? International Journal of Research in Marketing, 30(4), 421-423. https://doi.org/10.1016/j. ijresmar.2013.07.002

Kantar World Panel. (2017). El chocolate: la golosina de todas las edades Mexico - Kantar Worldpanel. https:// www.kantarworldpanel.com/mx/ Noticias-/El-chocolate-la-golosinade-todas-las-edades

Kaur, A., Lewis, T., Lipkova, V., Fernando, S., Rayner, M., Harrington, R. A., Waterlander, W., \& Scarborough,
P. (2020). A systematic review, and meta-analysis, examining the prevalence of price promotions on foods and whether they are more likely to be found on less-healthy foods. Public Health Nutrition, 8, 1281-1296. https://doi.org/10.1017/ S1368980019004129

Kim, H. M., \& Kramer, T. (2006). "Pay $80 \%$ " versus "get $20 \%$ off": The effect of novel discount presentation on consumers' deal perceptions. Marketing Letters, 17(4), 311-321. https://doi.org/10.1007/s11002-0069309-7

McCabe, S., \& Branco Illodo, I. (2019). Thrilled to Have "Bagged a Bargain" or "Bitter" and "Very Frustrating"? Exploring Consumer Attitudes to Value and Deals in Tourism. Journal of Travel Research, 58(6), 945-960. $\quad$ https://doi. org/10.1177/0047287518790403

McColl, R., Macgilchrist, R., \& Rafiq, S. (2020). Estimating cannibalizing effects of sales promotions: Theimpact of price cuts and store type. Journal of Retailing and Consumer Services, 53, 101982. https://doi.org/10.1016/j. iretconser.2019.101982

Mishra, A., \& Mishra, H. (2011). The influence of price discount versus bonus pack on the preference for virtue and vice foods. Journal of Marketing Research, 48(1), 196-206. https://doi.org/10.1509/jmkr.48.1.196

Morwitz, V. G., Greenleaf, E. A., \& Johnson, E. J. (1998). Divide and prosper: Consumers' reactions to partitioned prices. Journal of Marketing Research, 35(4), 453463. https://doi.org/10.2307/3152164

Park, S. (2016). Get Discount in Chocolate and Get More Toothpaste! The Effect of Product Preferences on Promotion About Price-Off and ValueAdded According to Product Type: 
Focusing on Hedonic and Utilitarian Product (pp. 275-280). Springer, Cham. https://doi.org/10.1007/978-3$319-26647-354$

Peng, L., Cui, G., \& Chung, Y. (2020). Do the pieces fit? Assessing the configuration effects of promotion attributes. Journal of Business Research, 109, 337-349. https://doi. org/10.1016/j.jbusres.2019.11.081

Petit, O., Lunardo, R., \& Rickard, B. (2020). Small is beautiful: The role of anticipated food waste in consumers' avoidance of large packages. Journal of Business Research, 113, 326-336. https://doi.org/10.1016/j. ibusres.2019.10.003

Purnhagen, K. P., \& van Herpen, E. (2017). Can Bonus Packs Mislead Consumers? A Demonstration of How Behavioural Consumer Research Can Inform Unfair Commercial Practices Law on the Example of the ECJ's Mars Judgement. Journal of Consumer Policy, 40(2), 217-234. https://doi.org/10.1007/s10603-0179345-0

Sinha, S. K., \& Verma, P. (2020). Impact of sales Promotion's benefits on perceived value: Does product category moderate the results? Journal of Retailing and Consumer Services, 52, 101887. https://doi.org/10.1016/j. iretconser.2019.101887
Smith, M. F., \& Sinha, I. (2000). The impact of price and extra product promotions on store preference. International Journal of Retail \& Distribution Management, 28(2), $\quad$ 83-92. https://doi. org/10.1108/09590550010315269

Valencia, M., Gustavo, R., Millán, G. \& Ulises, O. (2019). Tiendas Hard Discount y su incidencia en los minimercados boyacenses. Revista Venezolana de Gerencia, 127-143. https://doi.org/10.37960/revista. v24i2.31511

Vasić Nikcević, A., Alimpić, S., \& Perić, N. (2020). The efficiency of sales promotion methods emphasing the impulse behaviour: Case of Serbia. Quality - Access to Success, 21(175), 13-17.

Xu, Y., \& Huang, J.-S. (2014). Effects of Price Discounts and Bonus Packs on Online Impulse Buying. Social Behavior and Personality: an international journal, 42(8), 1293-1302. https://doi.org/10.2224/ sbp.2014.42.8.1293

Yu, A. P. I., Chuang, S. C., Cheng, Y. H., \& Wu, Y. C. (2020). The influence of sharing versus self-use on the preference for different types of promotional offers. Journal of Retailing and Consumer Services, 54, 102026. https://doi.org/10.1016/j. jretconser.2019.102026 